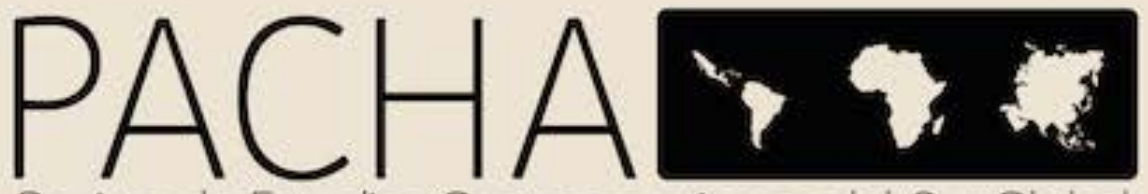

Revista de Estudios Contemporáneos del Sur Global Joumal of Contemporary Studies of the Global South Revista de Estudos Contemporâneos do Sul Global 


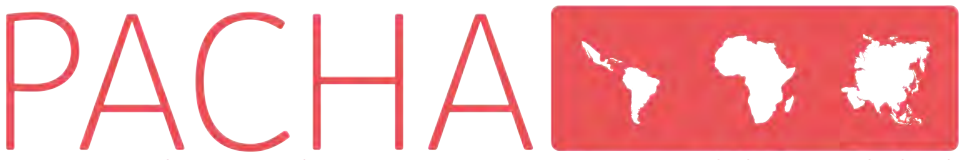

Revista de Estudios Contemporáneos del Sur Global

Joumal of Contemporary Studies of the Global South

\section{Equipo Editorial}

Carolina Díaz R.

Directora Editorial

CICSH-AL Centro de Investigaciones en Ciencias Sociales y Humanidades desde América Latina, Ecuador revistapacha@religacion.com; carolinadiaz@religacion.com

Roberto Simbaña Q.

Coordinador Editorial

CICSH-AL Centro de Investigaciones en Ciencias Sociales y Humanidades desde América Latina, Ecuador robertosimbana@religacion.com

\section{Editores Asociados}

Paola Andrea Tovar. Universidad De Montreal, Colombia. Editora Asociada en Antropología

Mitchell Alberto Alarcón Díaz. Universidad Nacional Mayor de San Marcos, Perú. Editor Asociado en Educación

Marcela Cristina Quinteros. Universidade Estadual de Maringá, Brasil. Editora Asociada en Historia Latinoamericana

Aboutaleb Sedatee Shamir. Islamic Azad University, Iran. Editor Asociado en Educación y Medio Oriente

Mateus Gamba Torres. Universidade de Brasilia - UNB, Brasil. Editor Asociado en Historia Mirna Yazmin Estrella Vega. Universidad Nacional Autónoma de México, México. Editora Asociada en Sociología

Rodrigo Navarrete Saavedra. Universidad Aus tral de Chile, Chile. Editor Asociado en Ciencias Políticas

Aygul Zufarovna Ibatova. Tyumen Industria University, Rusia. Editora Asociada en Humahidades sobre Asia
Shokhrud Fayziev Farmanovich. Tadqiqot, Tashkent, Uzbekistan. Editor Asociado en Desarrollo sobre la Comunidad de Estados Independientes, Asia.

Mª Aránzazu Serantes López. Woolf University, España. Editora Asociada en Humanidades digitales

Fabricio Espinosa Ortiz. Consejo Nacional de Ciencia y Tecnología, México. Editor Asociado en Geografía Humana

Marcelo Starcenbaum. Universidad Nacional de La Plata, Argentina. Editor Asociado en Filosofía e Historiografía

Siti Mistima Maat. Universiti Kebangsaan Malaysia, Malaysia. Editora Asociada en Innovación de aprendizaje

Carla Vanessa Zapata Toapanta. Universidad de Salamanca, España. Editora Asociada en Latinoamericanismo

\section{Consejo Editorial}

Andrea Paola Cantarelli, Universidad Nacional de Cuyo, Argentina
Federico Cabrera, Universidad Nacional de San Juan/CONICET, Argentina

Gamaniel David Suárez Cobix, Universidad Veracruzana, México

Gloria Concepción Tenorio Sepúlveda, Tecnológico de Estudios Superiores de Chalco, México

Héctor García Cornejo, Universidad Michoacana de San Nicolás de Hidalgo, México

Jorge Gilberto Bonilla Macas, Universidad Católica de Cuenca, Ecuador

José Alexander Rubiano Pedroza, Universidad de Pamplona, Colombia

María Dolores Sancho, Universidad Nacional del Comahue, Argentina

Mauricio Sandoval Cordero, Facultad Latinoamericana de Ciencias Sociales, Costa Rica

Rivera Varela Bertha Leticia, Universidad Abierta a Distancia, México

Tomás Sebastián Torres López, Universidad Alberto Hurtado, Chile

\section{CONSEJO ASESOR INTERNACIONAL}

\author{
Alejandro Mejía Tarazona \\ Facultad Latinoamericana de Ciencias Sociales, Ecuador \\ Alexander Luna Nieto \\ Fundación Universitaria de Popayán, Colombia \\ Celeste De Marco \\ CONICET/Universidad Nacional de Quilmes-CEAR, Argentina \\ Christian Andres Quinteros Flores \\ Universidad de Chile, Chile \\ Daniel Orizaga Doguim \\ Centro de Investigaciones Multidisciplinarias-UAQ, México \\ Francisco Javier Jover Mart \\ Universidad de Castilla-La Mancha, España
}

Gaya Makaran

Centro de Investigaciones sobre América Latina y el Caribe CIALC, UNAM, México

Jaime Araujo Frias

Universidad Nacional de San Agustín, Perú

João Luis Binde

Universidade Federal De Pernambuco, Brasil

Luisina Castelli Rodríguez

Universidad de la República, Uruguay

Mariana Jesica Lerchund

Universidad Nacional de Río Cuarto, Argentina

Marina Acosta

Universidad de Buenos Aires, Argentina

Noelia Marina Cortinas

Universidad de Buenos Aires, Argentin

Paulo Alves Pereira Júnior

Universidad Estatal Paulista, Brasil

Sergio Monroy Isaza

Universidad de Ibagué, Colombia

Suyai Malen Garcia Gualda

IPEHCS-CONICET-UNCO, Argentin 


\title{
Programa estratégico nacional para la correcta gestión de los residuos en México
}

\author{
National strategic program for the correct management of waste in Mexico
}

Sergio Javier Medina Peñaloza

Centro Universitario CIFE - México

sergiomedinape@gmail.com

\section{RESUMEN}

En este artículo se realiza un análisis documental de los principales instrumentos normativos en materia de gestión de los residuos a nivel internacional. Al respecto se exploran algunas de las prácticas en Italia, Noruega y Suecia; se comenta la situación actual de las políticas de gestión de los residuos en México; asimismo, de manera general, se identifican las principales causas por las que actualmente México carece de un programa estratégico nacional para la aplicación de la legislación existente, que permita el ejercicio práctico de los derechos y obligaciones inherentes, y se señalan las afectaciones fundamentales en el ambiente; finalmente, se plantea la necesidad de contar con un programa estratégico nacional para la adecuada gestión de los residuos en favor del uso racional y el desarrollo social sostenible.

Palabras clave: internacional; medio ambiente; México; nacional; separación de residuos.

\section{ABSTRACT}

This article performs a documentary analysis of the main regulatory instruments in the field of waste management at the international level. In this regard, some of the practices in Italy, Norway and Sweden are explored; the current situation of waste management policies in Mexico is discussed; likewise, in general, the main causes for which Mexico today lacks a strategic program for the application of existing legislation that allows the practical exercise of the inherent rights and obligations are identified, and are indicated the fundamental effects on the environment; finally, there is a need for a national strategic program for the proper management of waste in favor of the rational use and sustainable social development.

Keywords: environment; international; Mexico; national; waste separation. 


\section{Introducción}

En la actualidad, el ambiente es un tema de agenda obligado de los diversos gobiernos y organizaciones alrededor del mundo. Desde el siglo pasado, el cambio climático en nuestro planeta empezó a llamar la atención de los líderes mundiales, diplomáticos, empresarios, científicos, estudiosos y de la población en general (Luna-Nemecio, 2019); sus efectos repercutieron directamente en la salud de los seres humanos, de la flora y la fauna y de todos los recursos naturales; la tierra estaba enferma y pedía ayuda. El capitalismo, en su desaforada carrera contra nadie y contra todos, marcada por barreras, fronteras, diferencias, carencias morales y abundancia bélica, pretende ser dueño de un mundo del que no se ocupa y con el que no es capaz de relacionarse de manera respetuosa y responsable; $y$ al que, por el contrario de proteger y conservar, sobreexplota y destruye. La situación provocada evidentemente por nuestro comportamiento, se ha extendido a lo largo de las generaciones y de los territorios; se trata de un problema global. Ante esta realidad, hace algunos años, la Organización de las Naciones Unidas, entre otras instancias de orden internacional, empezaron a tomar partido en el combate de las afectaciones y reparación de los daños, así surgió el Derecho Internacional Ambiental, que es una disciplina del derecho creada para proteger desde el campo jurídico al ambiente.

Como es sabido el derecho ambiental surge por una preocupación universal, raíz de una toma de conciencia en los temas ecológicos, los cuales han sido bastante maximizados en los últimos tiempos. La comunidad internacional, claro, ha jugado un papel fundamental en el desarrollo de esta importante temática y de hecho ha tomado la delantera en el establecimiento de ciertos mecanismos para la protección del imperante recurso ambiental en cualquiera de sus formas. (Narváez, 2019, p. 372)

En la actualidad hablar del futuro sin implicaciones ambientales es prácticamente imposible; la importancia del Derecho Internacional Ambiental, y su aplicación en las prácticas sociales a nivel mundial es una necesidad de primer orden para la continuación de la vida y el progreso de la humanidad. Entre las principales causas del deterioro del planeta se encuentra la densificación demográfica de la población en espacios urbanos insustentables (Luna-Nemecio, 2017) y las cantidades gigantescas de residuos que se producen diariamente. En el caso de la erróneamente llamada basura, desde hace al menos diez años en todo el país se han redactado normas paliativas relativas a la importancia de la separación de los residuos que generalmente atienden aspectos conceptuales o terminológicos, pero hasta la fecha no se ha desarrollado un proyecto claro para atender el problema eficazmente, lo cual es grave para México, uno de los diez países más poblados del mundo, que cuenta con alrededor de 129 millones 163 mil habitantes.

Esto revela la necesidad urgente de generar estrategias y programas de atención de los residuos. Son pocos los países que han tomado medidas al respecto con base en la normatividad derivada de los trabajos de la Organización de las Naciones Unidas, a través de la autoridad especializada; los esfuerzos de la mayoría de los gobiernos por establecer programas específicos de protección ambiental avanzan lentamente, y en México, las políticas de gestión de los residuos son rudimentarias e insuficientes ante las exigencias de la actual y desalentadora realidad.

México ha firmado varios tratados y convenios internacionales en los cuales se compromete a procurar el desarrollo social sostenible (Luna-Nemecio, 2020), pero la incorrecta gestión de los residuos en el territorio nacional contribuye cotidianamente a la degeneración del medio ambiente, lo que da por resultado una contradicción evidente que urge subsanar, adecuando los compromisos adquiridos a la praxis nacional, no solamente desde el punto de vista de las obligaciones asumidas entre pares, sino también desde la perspectiva de la alta responsabilidad moral y humana que tenemos con el mundo y con las futuras generaciones.

\section{Principales instrumentos normativos a nivel internacional}

En el año de 1972, la Organización de las Naciones Unidas, creó el Programa de las Naciones Unidas para el Medio Ambiente que es el órgano que funge como autoridad a nivel mundial en temas relacionados con el ambiente, tiene su sede en Kenia, y trabaja por regiones: África, Asia y el Pacífico, América del Norte, Europa, Asia Occidental y América Latina y el Caribe. Su misión es trabajar en conjunto con todos los países del mundo para proteger el planeta, de modo que los actuales habitantes disfruten su estancia sin que ello signifique el detrimento de las oportunidades de las generaciones venideras; es decir, aprovechar nuestra estancia en la tierra asegurando un futuro saludable.

The United Nations Environment Programme (the UN Environment Programme) is the leading global authority on the environment. Since 1972, we are a powerful advocate, setting the global environmental agenda, providing leadership and delivering scientific solutions on some of the most urgent challenges facing 
the planet and humankind. [El Programa de las Naciones Unidas para el Medio Ambiente (Programa de las Naciones Unidas para el Medio Ambiente) es la principal autoridad mundial en materia de medio ambiente. Desde 1972, somos un poderoso defensor, estableciendo la agenda ambiental global, proporcionando liderazgo y entrega de soluciones científicas en algunos de los desafíos más urgentes que enfrenta el planeta y la humanidad]. (United Nations Environment Programme [UNEP], 2019, p. 4)

La Declaración de Estocolmo sobre el Medio Ambiente Humano es el primer gran documento en materia de Derecho Ambiental y fue el primero también en reconocer el derecho a un ambiente sano. (Gómez, 2014. P.125) Se gestó en el centro de una época controversial agitada por ideas con enfoque capitalista, donde la industrialización y su inercia se habían plasmado en la mente colectiva. El cuidado del ambiente constituía no solamente un interés común, sino una necesidad social de carácter global y urgente, sobrepasaba en importancia todo acontecimiento previo y condicionaba estrictamente el futuro. Su fundamento teleológico era el ser humano; el cuidado del ambiente se imponía contra cualquier capricho o resistencia a la unidad, el reconocimiento del otro como igual ya no bastaba, había que trabajar en equipo, concientizar la trascendencia del momento histórico que se vivía; enfrentar las consecuencias de los egos y ambiciones personales u organizacionales y sumarse sin demoras, a la reconstrucción del planeta, pues de ello dependían la existencia y la continuación de la vida.

En 1983, la Organización de las Naciones Unidas, creó la Comisión Mundial sobre el Medio Ambiente y el Desarrollo, también conocida como la Comisión Brundtland, cuya labor se centra en la relación entre ambiente y desarrollo. De esta Comisión, en abril de 1987, resultó el documento a modo de informe denominado Nuestro Futuro Común, en el cual se acuña por primera vez el concepto de desarrollo sostenible, mismo que hasta el momento constituye la base del Derecho Internacional Ambiental. "Está en manos de la humanidad asegurar que el desarrollo sea sostenible, es decir, asegurar que satisfaga las necesidades del presente sin comprometer la capacidad de las futuras generaciones para satisfacer las propias" (Gómez, s. f., p. 91).

Durante el mismo 1987, se firmó el Protocolo de Montreal, que se centra en la procuración de la restauración de la capa de ozono, con el objetivo de controlar las emisiones que la deterioran, considerando las necesidades particulares de los países en desarrollo. Asimismo se determina el establecimiento de mecanismos para identificar si los países firmantes cumplen o no con el tratado y poder actuar al respecto.

En virtud del convenio, las partes se comprometen a proteger la salud humana y el medio ambiente contra los efectos del agotamiento de la capa de ozono, y en dos anexos se estipula que los Estados participantes cooperarán en la investigación y el intercambio de información (Flores, 2015, p. 30).

En 1992, durante la celebración de la Cumbre de la Tierra en Río de Janeiro, se presentaron tres documentos: el Convenio sobre la Diversidad Biológica, la Convención Marco de las Naciones Unidas sobre el Cambio Climático y la Declaración de Río sobre el Medio Ambiente y el Desarrollo, todas ellas en atención del desarrollo sostenible.

En el 2016, México fue sede de la Conferencia de las Naciones Unidas sobre Biodiversidad para el Bienestar, que se llevó a cabo en Cancún, Quintana Roo, en ella se establece que: "Para México, ser un país megadiverso conllevaba la gran responsabilidad de cuidar su entorno, que no solo es patrimonio nacional, sino de toda la humanidad" (UNEP, 2016, p. 398).

La Convención Marco de las Naciones Unidas sobre el Cambio Climático se enfocó en disminuir los gases efecto invernadero y los países firmantes adquirieron, entre otros compromisos, el de informar periódicamente sobre sus planes de trabajo, las formas de cumplimiento, y los logros alcanzados en el periodo de tiempo correspondiente; en su mayoría, los compromisos asumidos tienden a la promoción y cooperación nacional e internacional para formular, elaborar y aplicar programas estratégicos que permitan lograr la meta propuesta.

La Declaración de Río sobre el Medio Ambiente y el Desarrollo celebrada en Río de Janeiro en junio de 1992, tuvo el objetivo de refrendar los compromisos asumidos en la Declaración de Estocolmo acerca de la urgencia de reparar los daños causados al ecosistema y uno de sus principales logros fue el compromiso que asumieron más de 190 países para adoptar el concepto de economía verde que tiene que ver específicamente con asuntos relacionados con el agua, la tierra y la biodiversidad. (Zarta, 2018, p. 422). 
Para 1994, la semilla de la conciencia acerca del cuidado del planeta empezaba a crecer, ejemplo de ello, es el Acuerdo de Marrakech, que da lugar a la creación de la Organización Mundial del Comercio y del que han surgido acciones de solidaridad con los países en desarrollo en cuanto a su financiación, apoyo en sus capacidades de adaptación; y en el desarrollo y transferencia de tecnología. Su objetivo principal es procurar una sana economía en observación del interés superior del cuidado del planeta, utilizando la producción conscientemente en favor de las buenas relaciones comerciales y el crecimiento económico mundial equitativo (Berruezo y Díaz, 2017).

Llegado el año 2000, surgió la Declaración del Milenio, que reconoce la necesidad de un crecimiento económico sostenible, y se suma a su precedente el Acuerdo Marrakech, esta vez enfocándose en los pobres y en la renovación del reconocimiento de los derechos humanos.

Pero, sobre todo, hay más claridad sobre la necesidad de que los pueblos puedan superar el miedo al futuro, logren dentro delo posible una mayor igualdad en el campo social y económico, alcancen el reconocimiento efectivo de sus derechos, puedan gozar de sus riquezas, mejoren los niveles de vida de la población, sean más competitivos, respetuosos del medio ambiente y actores dinámicos del progreso en el mundo globalizado. (Ramírez, 2010, “La Organización de las Naciones”, párr. 2)

En el año 2016, sale a la luz el Acuerdo de París, que dirige sus objetivos al mantenimiento de una temperatura saludable en el planeta; un documento normativo de especial importancia y realce por el impacto positivo que ha tenido en la realidad social, al ser base fundamental de la primera sentencia dictada en Inglaterra, mediante la cual se impidió la construcción de la ampliación de un aeropuerto; reconociendo con ello, el valor del compromiso de cada estado participante y respetando el derecho humano al desarrollo sostenible (Luna-Nemecio, 2020), traducido en un acto legal sin precedentes de motivaciones ambientales directas.

El 2017, fue un año revolucionario en el campo de las cortes judiciales, especialmente en el contexto de cuidado y reafirmación de la vida, así como de los derechos humanos. La Corte Interamericana de Derechos Humanos, siguiendo sus propias directrices y sumándose a las acciones en pro del medio ambiente, emitió la Opinión Consultiva sobre medio ambiente y derechos humanos, nombrada también Opinión Consultiva 23, reconociendo en ella, por primera vez, el derecho a un medio ambiente sano como fundamental para la existencia humana.

\footnotetext{
La Corte afirma el carácter autónomo de este derecho el que, en cuanto tal, protege los componentes del medio ambiente, tales como bosques, ríos, mares y otros. Asimismo, enfatiza que los daños ambientales pueden afectar todos los derechos humanos puesto que el disfrute de dichos derechos depende de la existencia de un medio propicio. También la Corte destacó la interdependencia e indivisibilidad que existe entre los derechos humanos, el medio ambiente y el desarrollo sostenible. (Sticca, 2018, p. 257)
}

América latina y el Caribe se vieron beneficiados con el Acuerdo de Escazú suscrito en 2018, instrumento a través del que se reconocen sus derechos de acceso a la información relacionada con el medio ambiente, a la participación pública y de acceso a la justicia.

Todos los documentos normativos en materia ambiental, independientemente de sus tópicos específicos, encaminan sus objetivos hacia el equilibrio ecológico y el desarrollo sostenible; se proponen alcanzar dichas metas a través de la planeación, desarrollo y aplicación de estrategias normadas, que exigen la participación y el apoyo de todos los países miembros de cada instrumento. De este modo, los Estados deben realizar su labor en el ámbito de sus respectivas jurisdicciones locales vinculando sus resultados y compartiendo sus experiencias inmediatas a través de las tecnologías existentes, haciendo uso del derecho a la información ambiental, reconocido por el propio Programa de las Naciones Unidas para el Medio Ambiente, como herramienta básica para una interacción constante, dinámica y eficaz, que facilite la labor entre pares y favorezca el progreso unánime.

Es sustancial que los países permanezcan firmes en los compromisos adquiridos y se mantengan motivados ante los embates y las complicaciones que impone la realidad o los contextos sociales, recordando 
que el fundamento original de sus esfuerzos y su trabajo es y será el mayor valor que debemos aquilatar, la vida misma. Tenemos una deuda con el planeta, somos responsables de asegurar un futuro saludable para el mundo y para todas las especies, y garantizar un horizonte de desarrollo para la humanidad.

\title{
Prácticas internacionales. Italia, Noruega y Suecia Italia
}

A partir de 1997, en Italia, derivado de la entrada en vigor del llamado Decreto Ronchi, la Ley Italiana se adecuó a la normatividad europea para legislar en materia ambiental; en el 2006 su normatividad nacional sobre los residuos sufrió una profunda transformación estableciendo medidas prioritarias para prevenir y reducir la producción de desperdicios, para lo que el incumplimiento de las reglas establecidas implica severas sanciones económicas que pueden ser aplicadas por varias instancias autorizadas, como policías estatales, municipales y por inspectores de empresas locales y regionales encargadas de la salud.

\begin{abstract}
La gerarchia di gestione de i rifiuti è disciplinata, in particolare, negli articoli da 179 a 182, dove coerentemente con la linea già definita dal Decreto "Ronchi", vengono stabilite quali misure prioritarie le azioni volte a prevenire e ridurre la produzione di rifiuti... [La jerarquía de gestión de residuos está regulada, en particular, en los artículos 179 a 182, donde en la línea ya definida por el decreto “Ronchi”, las acciones destinadas a prevenir y reducir la producción de residuos se establecen como medidas prioritarias...] (Salvioni, 2009, párr. 2).
\end{abstract}

Emanado de la adhesión de Italia a las normas de la Unión Europea para el cuidado del ambiente, y en relación con el paquete europeo de medidas sobre economía circular, se empezó a considerar a los residuos como potenciales recursos para crear sistemas sostenibles más eficientes que permitieran su optimización al utilizarlos como fuente generadora de energía para crear mercados económicos nuevos que apoyaran a los grupos más vulnerables. (Graziani, 2018, p. 50)

\begin{abstract}
Sólo en Italia se realizó la primera planta en el mundo para el reciclaje de pañales y toallas sanitarias por una multinacional propietaria de las marcas notoriamente conocidas, en colaboración con el gestor de residuos municipales Treviso (Contarina Spa), en colaboración con el Ayuntamiento Ponte en Alpes, el instituto de investigación Ambiente Italia, y con la cofinanciación de la Unión Europea en el marco del proyecto de Recall (Di Ciaula et al, 2015, p. 13).
\end{abstract}

Entre las prácticas relacionadas con la gestión de los residuos en Italia, podemos mencionar lo que ocurre en la provincia de Treviso, de la región del Véneto, donde cada contenedor de residuos cuenta con un chip asociado a la familia que corresponde y que permite calcular la cantidad de residuos que genera. Por otro lado, algunas personas suelen producir lo que se conoce como compost o composta en español, que es materia orgánica útil para el abono de las tierras; para ello, aprovechan los residuos orgánicos que obtienen después de separarlos. Esta práctica se convierte en un modo de conseguir beneficios económicos múltiples, pues al producir su propio fertilizante evitan adquirirlo a costos elevados, ahorrándose el pago que correspondería si desecharan la materia orgánica utilizada en su producción de compost, ya que la autoridad encargada de la recolección estima sus cuotas de acuerdo a la cantidad de residuos de los que la gente se deshace.

Los ejemplos de prácticas de prevención virtuosos están vendiendo productos de barril, la reintroducción de retornables (ampliamente utilizado en otros países) y, en cuanto a la fracción orgánica de los residuos sólidos urbanos (RSU), el compostaje doméstico (o auto compostaje), que, junto con la construcción de compostaje, se inscribe plenamente en las políticas de prevención (Di Ciaula et al, 2015, p. 6).

Estas prácticas muestran un nivel de comportamiento consiente e inteligente acorde con las necesidades, derechos y obligaciones de la actualidad ambiental. 


\title{
Noruega
}

Noruega es uno de los países con mayor compromiso y dedicación en el cuidado ambiental. Su primera normatividad sobre contaminación y desechos fue la denominada Ley de Control de la Contaminación de 1981, que contenía reglas básicas para el manejo de los residuos; para el año 2004 surgió una nueva regulación, que modificó la responsabilidad de los municipios, previamente su función abarcaba residuos de empresa y domésticos; actualmente incluye sólo estos últimos. Dentro de las prácticas Noruegas para la gestión de los residuos, se encuentra el Plastretur, un sistema que inició en el año 2002, centrado en la industria del plástico, con la misión de asegurar la recogida y recuperación del plástico a costos aceptables.

\begin{abstract}
Plastretur AS, the material company for plastic packaging, is responsible for the development and organization of collection and recovery to reach the goal in the covenant. According to the agreement, Plastretur is responsible for finding the solutions that reach the goals with the lowest possible costs. [Plastretur AS, la compañía de materiales para envases de plástico, es responsable del desarrollo y organización de la recolección y recuperación para alcanzar el objetivo del pacto. Según el acuerdo, Plastretur es responsable de encontrar las soluciones que alcanzan los objetivos con los costos más bajos posibles]. (Eik, et al, 2002, p. 39)
\end{abstract}

Oslo considera esencial la separación de los residuos para mantener una ciudad sana y limpia, así como para generar energía con la que proporcionan calefacción a sus escuelas y hogares, y que otras veces sirve para alimentar a las empresas encargadas de la propia gestión de los residuos, trabajan en ello, basados en lo que denominan la Próxima Revolución Industrial. "Next Industrial Revolution", where industry will be reshaped and where focus is on sustainable. ["Siguiente Revolución Industrial", donde la industria será remodelada y donde el enfoque es sostenible]" (Eik, et al, 2002, p.10). To make a more sustainable recycling system it is important to both reduce the use of energy in total and to shift from fossil fuels to renewable energy sources. [Para lograr un sistema de reciclaje más sostenible, es importante reducir el uso total de energía y pasar de los combustibles fósiles a las fuentes de energía renovables] (Eik, et al, 2002, p. 23).

En Noruega la basura doméstica es utilizada como combustible, como se dijo ya, esta energía es destinada principalmente a la calefacción que llega a las casas o instituciones públicas a través de maquinaria especializada para ello. Algunas ciudades de Reino Unido, llevan sus residuos a tratar a la ciudad de Oslo, lo que representa un ingreso adicional para Noruega (Yépez \& Viteri, 2019, p. 123).

Otra de las prácticas noruegas que favorecen al ambiente, es la producción de biogás a partir de residuos orgánicos, dos kilos de estos residuos producen aproximadamente un litro de bio-combustible que es utilizado para reducir costos en el área de transporte de sus ciudades (Yépez \& Viteri, 2019, p. 123). Oslo es, sin duda, pináculo en buenas y exitosas prácticas, ecológica y financieramente rentables.

\section{Suecia}

El Código Ambiental de Suecia es la base legal en materia ambiental para ese país; data de 1998 cuando fue creado y entró en vigor el año siguiente. Su objetivo primordial es promover el desarrollo sustentable para asegurar la salud ambiental de las presentes y futuras generaciones. Es aplicable en materia de reutilización y reciclaje, así como otros tipos de gestión de materiales, materias primas y energía con miras a establecer y mantener los ciclos naturales. La economía circular es la base de todas las prácticas suecas en favor del medio ambiente; es un enfoque que implica el uso de productos que pueden reutilizarse por completo. "La Economía Circular se presenta como una alternativa al actual modelo de producción y consumo, con el potencial de resolver retos medioambientales, al mismo tiempo que abre oportunidades de negocio y crecimiento económico" (Arroyo, 2018, p. 79).

El concepto de progreso que tiene Suecia, proviene de una concientización de la relación inherente entre el ser humano y el medio ambiente, un hecho que a pesar de su obviedad pasa muchas veces desapercibido, y que se inculca a los niños suecos desde edades tempranas, enseñándoles que el mundo que los rodea forma parte de ellos, que los recursos no son ilimitados y que la base de la paz es el respeto.

Como parte de la economía circular Suecia recicla y al mismo tiempo, de manera similar al caso de Noruega, produce energía a partir de sus residuos orgánicos mediante un programa establecido especialmente para ello, "el país europeo apostó por el reciclaje, su población se caracteriza por reciclar el $99 \%$ de sus residuos orgánicos y el $88 \%$ de los inorgánicos y es así como ahora produce energía a través 
del programa "De desecho a energía" (Arroyo, 2018, p. 94).

Para muchos de estos países, el presente es un tiempo de actuar con eficacia y creatividad para vislumbrar un futuro. ¿Qué esfuerzos ha hecho México?

\section{Situación actual de la gestión de los residuos en México}

Los instrumentos internacionales referidos en el presente artículo son la base normativa internacional que tiene México en materia ambiental, y que fungen simultáneamente, como pauta y obligación de su participación activa en el logro de los objetivos globales. A nivel nacional, en materia de derecho ambiental, México cuenta con varias disposiciones legales, entre ellas, la Ley General para la Prevención y Gestión Integral de Residuos; a través de la cual los Municipios tienen la función del manejo los residuos, consistente en recolectarlos, trasladarlos y tratarlos hasta su disposición final, todo ello debería llevarse a cabo mediante la formulación de programas para gestión de los residuos, comúnmente llamados basura; sin embargo, la realidad es que los programas comentados no existen y los residuos carecen de un tratamiento adecuado.

Actualmente los pasos de México a nivel nacional en la procuración del medio ambiente, particularmente en la atención de los residuos, son dirigidos por las disposiciones siguientes:

El Título Segundo de la Ley General para la Prevención y Gestión Integral de los Residuos, que versa sobre la distribución de competencias, en su artículo 7 fracción XXVIII establece como facultad de la Federación el convocar a entidades federativas y municipios para el desarrollo de estrategias conjuntas en materia de residuos que permitan la solución de los problemas que los afecten; por su parte, el artículo 26 de la referida Ley determina que las entidades federativas y los municipios deberán elaborar e instrumentar los programas necesarios para gestionar adecuadamente los residuos.

México es una República representativa formada por Estados libres y soberanos en su régimen interior, al igual que Argentina y Brasil; por lo que el manejo de los residuos, tema de gran importancia, se deja en manos de cada uno de los 32 Estados en que se divide administrativamente el país; pero constitucionalmente la actividad y obligación de recolectar basura, connotación muy empleada, se delega en las administraciones municipales.

Pondré un ejemplo. Uno de los 32 estados que conforman la República es el Estado de México, con una población aproximada de 17 millones de habitantes y subdividido políticamente, a su vez, en 125 municipios a manera de administraciones o ayuntamientos provinciales, que tienen dentro de sus atribuciones la obligación de prestar el servicio de recolección de residuos. Su reglamentación se encuentra en los Ilamados "Bandos Municipales", y concretamente, el municipio de mi residencia, llamado Toluca, en su Bando Municipal 2020, se refiere como obligación de los vecinos del municipio, entregar sus residuos sólidos domésticos debidamente separados al personal de los vehículos recolectores; así mismo, se señala que el municipio prestará como servicio público la limpia y tratamiento integral de residuos sólidos; también se atribuye a la autoridad municipal prevenir y controlar la contaminación del suelo por residuos sólidos domésticos para lo cual se deben aplicar disposiciones jurídicas correspondientes.

El problema en México, en el campo del Derecho Ambiental y del tratamiento de los residuos, no es sólo la carencia legislativa homologada a nivel nacional, sino la ausencia de estrategias que permitan hacer factible la aplicación de todas las disposiciones existentes; y el ejercicio práctico de los derechos y obligaciones inherentes. En México, existe un rico bagaje legislativo en materia ambiental; normas internas que siguen las líneas de regulaciones internacionales, en su mayoría propuestas por los gobiernos más avanzados en desarrollo sostenible, quienes a través del cumplimiento de las disposiciones han logrado objetivos trascendentales en la restauración del medio ambiente; y que impactan en la realidad colectiva; lamentablemente, los resultados alcanzados por naciones extranjeras y por México, siguen siendo insuficientes para la capitalización de los objetivos exclusivamente nacionales y las metas comunes de los países.

\section{Causas por las que México carece de un programa estratégico nacional para la cor- recta gestión de los residuos y sus afectaciones al medio ambiente}

México es un país que en cada espacio de tiempo continúa escribiendo su destino desde su legado de cultura y tradiciones. Es considerado como un país megadiverso porque posee casi el setenta por ciento de la diversidad mundial de especies (animales y plantas), con litorales tanto en el Atlántico, como en el Pacífico, que se impregna de una abundante historia y una cultura rica en tradiciones reconocidas en el mundo. Pero no ha llegado aún a consolidar una disciplina sólida que genere positivos cambios sociales a través de la maravillosa herramienta que representa el Derecho Ambiental. Padece del mismo mal que 
muchos otros países en vías de desarrollo: adolece de conciencia ambiental y de reconocerse parte de un complejo y basto mundo cuya subsistencia está destinada a preservar su ambiente, no sólo a través de la persuasión y obligatoriedad de las normas, sino con acciones concretas, de espectro nacional y permanentes.

De acuerdo a lo anterior y a lo expresado en este texto, me atrevo a realizar una contundente afirmación: México carece de un programa estratégico nacional para la correcta gestión de los residuos, que no puede dejarse legalmente sólo como una obligación de las administraciones provinciales municipales.

Aun cuando los mayores esfuerzos por atender este problema se han hecho en el ámbito local municipal, por un lado, falta un blindaje de los proyectos ambientales a través de políticas públicas nacionales; y por otro, deben trascender a las administraciones, considerando que los ayuntamientos municipales se cambian cada tres años y las estatales sexenalmente, pero en todo caso, no existe continuidad en las incipientes políticas ambientales.

En lo que toca a la conciencia social de separación de los residuos, el crecimiento poblacional exponencial, la ignorancia de las normas, la incultura ecológica, la carencia de educación ambiental, el descuido de los valores, el desarraigado sentido de pertenencia social, hace que diariamente los porcentajes de residuos separados sean los mínimos, mientras que la mayor parte de ellos se desechan sin tratamiento previo, pues para la recolección no se exige al ciudadano la separación de residuos ni se prevé una política regulatoria sancionadora o premial por el incumplimiento o cumplimiento de tal separación, según sea el caso.

A nivel gubernamental, pudiera enunciarse la insuficiente comunicación entre instituciones, el mínimo trabajo en equipo, desequilibrio en la distribución de los recursos económicos, ineficaz asignación de facultades decisivas, inhabilidad estratégica, y erróneas interpretaciones de la ley. También cuentan entre las causas: la lucha contra el tiempo, la necesidad de aprobación jerárquica y la dificultad para establecer consensos y metas comunes. Todo lo cual afecta e interrumpe el cumplimiento de los compromisos asumidos en los foros internacionales; consintiendo tácitamente el deterioro continuado del medio ambiente y los correspondientes impactos en la salud de los seres humanos, lo que propicia innegables retrocesos en la escalada mundial del Derecho Ambiental.

\begin{abstract}
La escasez de recursos financieros, humanos y tecnológicos son un obstáculo frecuente para la buena gestión de los residuos y la implementación de tecnologías innovadoras. No obstante, se puede mejorar notablemente y contribuir a ciudades más limpias, saludables y sostenibles ambientalmente con una mayor planificación y con estrategias encaminadas a cubrir todos los aspectos del ciclo de los residuos (ONU-HABITAT, 2012) (Graziani, 2018, p. 16).
\end{abstract}

Para concluir, vale la pena señalar que aunque se tuviera una regulación adecuada y de avanzada, es menester generar en el aspecto social, tanto individual como grupal, una férrea conciencia ambiental, entendida como una filosofía existencial de preocupación por la conservación del medio ambiente, que puede iniciar con decisiones sencillas que acarrean grandes pasos: la convencida y voluntaria separación de residuos en cada hogar para su posterior tratamiento y, en esa medida, exigir como ciudadano que se emule el ejemplo de países desarrollados, que poseen altos niveles de calidad de vida no sólo por su índice per cápita y su economía, sino sobre todo por el elevado índice de conciencia social y desarrollo humano para tener una vida saludable y un ambiente seguro. ¿Qué tanto contribuyes tú desde tu espacio a lograrlo?

\title{
Conclusiones
}

Es necesario contar en México con un Plan Estratégico Nacional que regule la adecuada gestión de los residuos en favor del uso racional y el desarrollo sostenible del medio ambiente.

El Derecho Ambiental en México debe tener una atención prioritaria y transversal indispensable en los proyectos de trabajo de todas las administraciones, sean Federales, Estatales o Municipales y debe regularse en el derecho nacional constitucional como parte del "derecho a una vida digna", para que trascienda administraciones con efectos permanentes.

Las políticas de los distintos órdenes de gobierno deben alinearse a los tratados suscritos por México en materia de medio ambiente, para que la recolección de residuos no sólo quede como obligación de las administraciones municipales, sino como una política nacional de protección al medio ambiente, estableciendo consecuencias administrativas por su incumplimiento. 


\section{Conflict of interest}

No potential conflict of interest is reported by the author(s).

\section{Funding}

There is no financial assistance in studies from external parties.

\section{Acknowledgements}

$\mathrm{N} / \mathrm{A}$

\section{REFERENCIAS}

Arroyo, F. (2018). La economía circular como factor de desarrollo sustentable del sector productivo. INNOVA Research Journal, (3)12, 78-98. https://doi.org/10.3389o/innova.v3.n12.2018.786

Berruezo. A., y Díaz J. (2017) Situación del Convenio Marco de Naciones Unidas sobre el Cambio Climático. Resumen de las Cumbres de París, COP21 y de Marrakech, COP22. Revista de Salud Ambiental. 17(1), 34-39. https://bit.ly/35iiVod

Di Ciaula, A., Gentilini, P., Laghi, F., \& Migaleddu, V. (2015, 12 de agosto). La gestión sostenible de la basura sólidos urbanos. International Society of Doctors for Environment. https://bit.ly/3d7311Z

Eik, A., Skeinmo, S., Solem, H., Brattebo, H., \& Saugen, B. (2002). Eco-Efficiency in Recycling Systems. Evaluation Methods \& Case Studies for Plastic Packaging. Norwegian University of Science and Technology (NTNU)-Industrial Ecology Programme (IndEcol). https://bit.ly/35jVDXV

Flores, F. J. (2015, enero-junio). El protocolo de Montreal relativo a las sustancias que agotan la capa de ozono (1999) y la enmienda de Beijing. Un análisis de sus contenidos, sus alcances y sus repercusiones presentes. Revista Relaciones Internacionales, 88.1, 27-48. https://www.revistas.una. ac.cr/index.php/ri/article/view/6661

Gómez, C. (s. f.). III. El desarrollo sostenible: conceptos básicos, alcance y criterios para su evaluación. [Capítulo de libro alojado en el portal de la UNESCO]. https://bit.ly/3bOr1QE

Gómez, J. L. (2014, enero-junio). Del desarrollo sostenible a la sustentabilidad ambiental. Revista Facultad de Ciencias Económicas: Investigación y Reflexión, 22(1), 115-136. https://www.redalyc.org/articulo. oa?id=90931814009

Graziani, P. (2018). Economía circular e innovación tecnológica en residuos sólidos: Oportunidades en América Latina. CAF. Banco de Desarrollo de América Latina. https://bit.ly/zaQn7Wo

Luna-Nemecio, J. (2017). La insustentabilidad socioambiental de la producción del espacio urbano en el capitalismo Eepecíficamente neoliberal. Revista de Geografía Espacios, 6(11), 89-109. https://doi. org/10.25074/07197209.11.609

Luna-Nemecio, J. (2019). Calentamiento global y conflictividad sociohídrica ante el colapso ambiental del estado de Morelos, México. RELEG. Revista Latinoamericana de Estudiantes de Geografía, (6), 74-84. https://releg.org/pdf/releg2019n6_pp74-84.pdf

Luna-Nemecio, J. (2020). Para pensar el desarrollo social sostenible: múltiples enfoques, un mismo objetivo. Mount Dora (USA)/Quito (Ecuador): Kresearch/ Religación. Centro de Investigaciones en Ciencias Sociales y Humanidades desde América Latina. https://doi.org/10.35766/dss20

Narváez, A., (2019). Los principios del derecho internacional ambiental: evolución y aplicación en la sentencia stc-4360 del 2018 de la Corte Suprema de Justicia Colombiana. Derecho y cambio social, (56), 370-387. https://bit.ly/2KKSvuP

Ramírez, G. (2010). La declaración del milenio naturaleza, principios y valores. Oasis, 15. https://bit.ly/2YiUj5Z

Salvioni, E., (2009). Normativita Nazionale sui rifiuti. Eso Società Benefit Arl, Società Specializzata Nella Gestione Rifiuti. https://bit.ly/3cYyNrp

Sticca, M. (2018). Medio ambiente y Derechos Humanos. Opinión consultiva de la Corte Interamericana de Derechos Humanos OC 23/17 del 15 de noviembre de 2017 - Solicitada por la República de Colombia. Revista De La Facultad De Derecho, 9(1), 253-260. https://revistas.psi.unc.edu.ar/index. php/refade/article/view/24423 
United Nations Environment Programme. (2016, 7 de diciembre). Convenio sobre la diversidad biológica. CBD/ $\mathrm{COP} / 13 / 25$. https://bit.ly/2KL25O3

United Nations Environment Programme. (2019). Invest in a Healthy Planet-Invest in UN Environment. https:// bit.ly/2YjJoL5

Yépez. A., \& Viteri. F. (2019). Innovative approaches of the environ mental education with the use of urban organic residues. Revista Cátedra, 2(2), 111-132. https://doi.org/10.29166/catedra.v2i2.1639

Zarta, P. (2018). La sustentabilidad o sostenibilidad: un concepto poderoso para la humanidad. Tabula Rasa, 28, 409-423. https://doi.org/10.25058/20112742.n28.18

\section{Author:}

Sergio Javier Medina Peñaloza. Doctor en Derecho y Doctor en Ciencias Penales y Política Criminal. Doctorante en Socioformación y Sociedad del Conocimiento en el Centro Universitario CIFE. Actualmente es Magistrado en retiro del Tribunal Superior de Justicia. Poder Judicial del Estado de México 